\title{
Neutrino mass textures with one vanishing minor and two equal cofactors
}

\author{
Weijian Wang ${ }^{1,2}$ \\ ${ }^{1}$ Department of Physics, North China Electric \\ Power University, Baoding 071003, P. R. China \\ ${ }^{2}$ Zhejiang Institute of Modern Physics, \\ Zhejiang University, Hangzhou 310027, P.R. Chind*
}

\begin{abstract}
In this paper, we carry out a numerical and systematic analysis of the neutrino mass textures, which contain one vanishing minor and an equality between two cofactors. Among 60 logically possible textures, only eight of them are excluded for both normal and inverted hierarchy by the current experimental data at $3 \sigma$ level. We also demonstrate that the future long-baseline neutrino oscillation experiments, especially for the measurement of $\theta_{23}$ mixing angle, will play the important role in the model selection. The phenomenological implications from neutrinoless double beta decay and the cosmology observation are also examined. A discussion on the flavor symmetry realization of the textures is also given.
\end{abstract}

*Electronic address: wjnwang96@gmail.com 


\section{INTRODUCTION}

By this time, neutrino oscillation experiments have provided us with convincing evidences for massive neutrinos and leptonic flavor mixing. The bi-large flavor structure with two large mixing angle $\left(\theta_{12} \approx 34^{\circ}, \theta_{23} \approx 38^{\circ}\right)$ and the mass-squared differences $\left(\delta m^{2} \equiv m_{2}^{2}-m_{1}^{2}\right.$ and $\left.\Delta m^{2} \equiv\left|m_{3}^{2}-\left(m_{1}^{2}+m_{2}^{2}\right) / 2\right|\right)$ have been revealed by solar, atmosphere and accelerator oscillation experiments with high degree of accuracy [1] . Recently, the third mixing angle $\theta_{13}$ has been precisely measured by Daya Bay[2] and RENO[3] experiments. The relative large value of $\theta_{13}\left(\approx 9^{\circ}\right)$ opens the door for us to explore the leptonic CP violation and determine the neutrino mass hierarchy in the future long-baseline oscillation experiments. Although the absolute mass scale and the type of neutrino (Dirac type or Majorana type) is still unknown, they are constrained by the cosmology observation [4, 5] and neutrinoless double-beta decay experiments (for a review, see [6]). From the point of model building, it is important to find the appropriate textures of leptonic mass matrices consistent with the current experimental data. Many ideas have been made to explore the phenomenologically acceptable textures, such as texture zeros [7], hybrid textures [8, 9], zero trace[10], zero determinant [11], vanishing minors [12], two traceless submatrices[13] Recently the patterns with two equalities between elements and cofactors [14] have been investigated in $3 \times 3$ neutrino mass textures. Furthermore, it is pointed that some of the patterns, not all of them though, can be naturally realized by introducing proper flavor symmetry in the framework of seesaw mechanism.

In this paper, we systematically study the pattern of $M_{\nu}$ with one vanishing minor and an equality between cofactors. In the type-I seesaw model[15], the effective neutrino mass matrix is given by $M_{\nu} \approx M_{D} M_{R}^{-1} M_{D}^{T}$ where $M_{D}$ and $M_{R}$ are the Dirac neutrino mass and right-handed Majorana neutrino mass matrix respectively. In the basis where $M_{D}$ is diagonal, the vanishing minor in $M_{\nu}$ is equivalent to the zero textures of $M_{R}\left[16\right.$. If $M_{D}$ is assumed to be proportional to unit matrix, as having been made in Ref[14], an equality between two cofactors can be seen as two equal elements in $M_{R}$. However, as we will shown in the following section, in some cases 
the unit $M_{D}$ condition can be loosen to the diagonal matrix with two unit elements. Anyway, we are effectively studying the cases with one zero element and two equal elements in $M_{\nu}^{-1}$. In the following, we assume the neutrinos to be majorana particles where the mass matrix $M_{\nu}$ is a $3 \times 3$ complex symmetric matrix. If one of elements of $M_{\nu}^{-1}$ is zero and two of the others are equal, we have $C_{6}^{1} \cdot C_{5}^{2}=60$ logically possible patterns.

The plan of the paper is organized as follows: in Sec. II, we show standard formation for the three-flavor neutrino mixing and its link to the experimental results. The numerical results are given in Sec. III. We summary our results in Sec. IV, where a discussion on flavor symmetry realization is also given.

\section{FORMALISM}

\section{A. Classification of The 60 Patterns}

As having mentioned, we have 60 logically possible textures for the mass matrix with one vanishing minor and two equal cofactor. Thus, it is helpful for us to classify and label these patterns. According to the zeros in the $M_{\nu}^{-1}$, we classified the textures 
into six subgroups A-F as following

$$
\begin{array}{lll}
A 1: C_{11}=0, C_{12}=C_{13} ; & A 2: C_{11}=0, C_{12}=C_{22} ; & A 3: C_{11}=0, C_{13}=C_{23} ; \\
A 4: C_{11}=0, C_{22}=C_{23} ; & A 5: C_{11}=0, C_{22}=C_{33} ; & A 6: C_{11}=0, C_{23}=C_{33} ; \\
A 7: C_{11}=0, C_{12}=C_{23} ; & A 8: C_{11}=0, C_{13}=C_{33} ; & A 9: C_{11}=0, C_{13}=C_{22} ; \\
& A 10: C_{11}=0, C_{12}=C_{33} ; & \\
B 1: C_{12}=0, C_{11}=C_{13} ; & B 2: C_{12}=0, C_{13}=C_{22} ; & B 3: C_{12}=0, C_{13}=C_{23} ; \\
B 4: C_{12}=0, C_{13}=C_{33} ; & B 5: C_{12}=0, C_{11}=C_{22} ; & B 6: C_{12}=0, C_{11}=C_{23} ; \\
B 7: C_{12}=0, C_{11}=C_{33} ; & B 8: C_{12}=0, C_{22}=C_{23} ; & B 9: C_{12}=0, C_{22}=C_{33} ; \\
& B 10: C_{12}=0, C_{23}=C_{33} ; & \\
C 1: C_{13}=0, C_{11}=C_{12} ; & C 2: C_{13}=0, C_{11}=C_{22} ; & C 3: C_{13}=0, C_{11}=C_{23} ; \\
C 4: C_{13}=0, C_{11}=C_{33} ; & C 5: C_{13}=0, C_{12}=C_{22} ; & C 6: C_{13}=0, C_{12}=C_{23} ; \\
C 7: C_{13}=0, C_{12}=C_{33} ; & C 8: C_{13}=0, C_{22}=C_{23} ; & C 9: C_{13}=0, C_{22}=C_{33} ; \\
& C 10: C_{13}=0, C_{23}=C_{33} ; & \\
D 1: C_{22}=0, C_{11}=C_{12} ; & D 2: C_{22}=0, C_{11}=C_{13} ; & D 3: C_{22}=0, C_{11}=C_{23} ; \\
D 4: C_{22}=0, C_{11}=C_{33} ; & D 5: C_{22}=0, C_{12}=C_{13} ; & D 6: C_{22}=0, C_{12}=C_{23} ; \\
D 7: C_{22}=0, C_{12}=C_{33} ; & D 8: C_{22}=0, C_{13}=C_{23} ; & D 9: C_{22}=0, C_{13}=C_{33} ; \\
E 1: C_{23}=0, C_{11}=C_{12} ; & E 2: C_{23}=0, C_{11}=C_{13} ; & E 3: C_{23}=0, C_{11}=C_{22} ; \\
E 4: C_{23}=0, C_{11}=C_{33} ; & E 5: C_{23}=0, C_{12}=C_{13} ; & E 6: C_{23}=0, C_{12}=C_{22} ; \\
E 7: C_{23}=0, C_{12}=C_{33} ; & E 8: C_{23}=0, C_{13}=C_{22} ; & E 9: C_{23}=0, C_{13}=C_{33} ; \\
& D 10: C_{22}=0, C_{23}=C_{33} ; &
\end{array}
$$

$F 1: C_{33}=0, C_{11}=C_{12} ; \quad F 2: C_{33}=0, C_{11}=C_{13} ; \quad F 3: C_{33}=0, C_{11}=C_{22} ;$

$F 4: C_{33}=0, C_{11}=C_{23} ; \quad F 5: C_{33}=0, C_{12}=C_{13} ; \quad F 6: C_{33}=0, C_{12}=C_{22}$;

$F 7: C_{33}=0, C_{12}=C_{23} ; \quad F 8: C_{33}=0, C_{13}=C_{22} ; \quad F 9: C_{33}=0, C_{13}=C_{23} ;$

$F 10: C_{33}=0, C_{22}=C_{23}$; 
where the $C_{i j}$ denotes the cofactor of element $(i, j)$ in $3 \times 3$ neutrino mass matrix. For example, for the A1 texture with the condition $C_{11}=0, C_{12}=C_{13}$, we have

$$
M_{22} M_{33}-M_{23} M_{32}=0
$$

and

$$
(-1) \cdot\left(M_{21} M_{33}-M_{23} M_{31}\right)-\left(M_{21} M_{32}-M_{22} M_{31}\right)=0
$$

On the other hand, we can also classify the textures in the formation of $M_{\nu}^{-1}$, with one zero and two equal elements. Then the A1 pattern, for example, can denote as

$$
\left(\begin{array}{ccc}
0 & \triangle & \triangle \\
\triangle & \times & \times \\
\triangle & \times & \times
\end{array}\right)
$$

where the " $\triangle$ " stand for the nonzero and equal elements, while the " $\times$ " stand for arbitrary elements. The type of the matrix like (41) has appeared in the so-called hybrid neutrino mass texture[8]. However, the situation here is totally different where the hybrid feature shows not in $M_{\nu}$ but in $M_{\nu}^{-1}$; thus we can call the texture given in (11) the hybrid $M_{\nu}^{-1}$ textures.

\section{B. Standard Notation and Important Relations}

In this section, we construct the neutrino mass texture in terms of three neutrino mass eigenvalues $\left(m_{1}, m_{2}, m_{3}\right)$, three mixing angles $\left(\theta_{12}, \theta_{23}, \theta_{13}\right)$ and three CPviolating phase $(\delta, \alpha, \beta)$. In the basis where the charged mass matrix is diagonal, the neutrino mass texture $M_{\nu}$ under flavor basis is given by[17]

$$
M_{\nu}=P_{l} V M_{\text {diag }} V^{T} P_{l}
$$

where $M_{\text {diag }}$ is the diagonal matrix of neutrino mass eigenvalues $M_{\text {diag }}=\operatorname{diag}\left(m_{1}, m_{2}, m_{3}\right)$ and $P_{l}$ denotes the phases which are unobservable and depend on phase convention

$$
P_{l}=\left(\begin{array}{ccc}
e^{i \phi_{e}} & 0 & 0 \\
0 & e^{i \phi_{\mu}} & 0 \\
0 & 0 & e^{i \phi_{\tau}}
\end{array}\right)
$$


The Pontecorvo-Maki-Nakagawa-Sakata matrix [18] $V$ can be parameterized as $V=$ $U P_{\nu}$ with

$$
U=\left(\begin{array}{ccc}
c_{12} c_{13} & c_{13} s_{12} & s_{13} e^{-i \delta} \\
-s_{12} c_{23}-c_{12} s_{13} s_{23} e^{i \delta} & c_{12} c_{23}-s_{12} s_{13} s_{23} e^{i \delta} & c_{13} s_{23} \\
s_{23} s_{12}-c_{12} c_{23} s_{13} e^{i \delta} & -c_{12} s_{23}-c_{23} s_{12} s_{13} e^{i \delta} & c_{13} c_{23}
\end{array}\right)
$$

and

$$
P_{\nu}=\left(\begin{array}{ccc}
1 & 0 & 0 \\
0 & e^{i \alpha} & 0 \\
0 & 0 & e^{i(\beta+\delta)}
\end{array}\right)
$$

Here the abbreviation $s_{i j}=\sin \theta_{i j}$ and $c_{i j}=\cos \theta_{i j}$ is used. The $\alpha$ and $\beta$ in $P_{\nu}$ denote two Majorana CP-violating phases and $\delta$ denotes the Dirac CP-violating phase. In neutrino oscillation experiments, CP violation effect is usually reflected by the Jarlskog rephasing invariant quantity[19] defined as

$$
J \equiv \operatorname{Im}\left(U_{e 1} U_{\mu 2} U_{e 2}^{*} U_{\mu 2}^{*}\right)=s_{12} s_{23} s_{13} c_{12} c_{23} c_{13}^{2} \sin \delta
$$

Using (5) , any element $M_{\nu(a b)}$ in the neutrino mass matrix can be expressed as

$$
M_{\nu(a b)}=e^{i\left(\phi_{a}+\phi_{b}\right)} \sum_{i=1}^{3} V_{a i} V_{b i} m_{i}
$$

The cofactors of $M_{\nu}$ are

$$
\begin{gathered}
(-1)^{m+n}\left(M_{\nu(a b)} M_{\nu(c d)}-M_{\nu(e f)} M_{\nu(g h)}\right) \\
=(-1)^{m+n} \sum_{i, j=1}^{3}\left(e^{i\left(\phi_{a}+\phi_{b}+\phi_{c}+\phi_{d}\right)} V_{a i} V_{b i} V_{c j} V_{d j}-e^{i\left(\phi_{e}+\phi_{h}+\phi_{g}+\phi_{h}\right)} V_{e i} V_{f i} V_{g j} V_{h j}\right) m_{i} n(21 j 1)
\end{gathered}
$$

where $m$ and $n$ refer to the cofactor $C_{m n}$. It is observed that for any cofactor there is an inherent property

$$
\phi_{a}+\phi_{b}+\phi_{c}+\phi_{d}=\phi_{e}+\phi_{h}+\phi_{g}+\phi_{h}
$$

Thus we can extract this total phase factor from the bracket in Eq. (11).

The textures we concerned have the property

$$
M_{\nu(p q)} M_{\nu(r s)}-M_{\nu(t u)} M_{\nu(v w)}=0
$$


corresponding to the vanishing minor condition and

$$
(-1)^{m+n}\left(M_{\nu(a b)} M_{\nu(c d)}-M_{\nu(e f)} M_{\nu(g h)}\right)-(-1)^{m^{\prime}+n^{\prime}}\left(M_{\nu\left(a^{\prime} b^{\prime}\right)} M_{\nu\left(c^{\prime} d^{\prime}\right)}-M_{\nu\left(e^{\prime} f^{\prime}\right)} M_{\nu\left(g^{\prime} h^{\prime}\right)}\right)=0
$$

corresponding to the equivalent cofactor condition. Using (10), (11) and the intrinsic property (12), we obtain two equations for vanishing minor condition and equivalent cofactor condition, i.e

$$
\begin{aligned}
& m_{1} m_{2} K_{3} e^{2 i \alpha}+m_{2} m_{3} K_{1} e^{2 i(\alpha+\beta+\delta)}+m_{3} m_{1} K_{2} e^{2 i(\beta+\delta)}=0 \\
& m_{1} m_{2} L_{3} e^{2 i \alpha}+m_{2} m_{3} L_{1} e^{2 i(\alpha+\beta+\delta)}+m_{3} m_{1} L_{2} e^{2 i(\beta+\delta)}=0
\end{aligned}
$$

where

$$
\begin{gathered}
K_{i}=\left(U_{p j} U_{q j} U_{r k} U_{s k}-U_{t j} U_{u j} U_{v k} U_{w k}\right)+(j \leftrightarrow k) \\
L_{i}=(-1)^{m+n} Q\left(U_{a j} U_{b j} U_{c k} U_{d k}-U_{e j} U_{f j} U_{g k} U_{h k}\right) \\
-(-1)^{m^{\prime}+n^{\prime}}\left(U_{a^{\prime} j} U_{b^{\prime} j} U_{c^{\prime} k} U_{d^{\prime} k}-U_{e^{\prime} j} U_{f^{\prime} j} U_{g^{\prime} k} U_{h^{\prime} k}\right)+(j \leftrightarrow k)
\end{gathered}
$$

with $(i, j, k)$ a cyclic permutation of $(1,2,3)$ and $Q$ defined as

$$
Q \equiv e^{i \Phi}=e^{i\left(\phi_{a}+\phi_{b}+\phi_{c}+\phi_{d}-\phi_{a^{\prime}}-\phi_{b^{\prime}}-\phi_{c^{\prime}}-\phi_{d^{\prime}}\right)}
$$

After solving Eq.(15) and (16), we arrive at

$$
\begin{gathered}
\frac{m_{1}}{m_{2}} e^{-2 i \alpha}=\frac{K_{3} L_{1}-K_{1} L_{3}}{K_{2} L_{3}-K_{3} L_{2}} \\
\frac{m_{1}}{m_{3}} e^{-2 i \beta}=\frac{K_{2} L_{1}-K_{1} L_{2}}{K_{3} L_{2}-K_{2} L_{3}} e^{2 i \delta}
\end{gathered}
$$

With the help of Eq.(20) and (21), we obtain the magnitudes of mass radios

$$
\begin{aligned}
\rho & =\left|\frac{m_{1}}{m_{3}} e^{-2 i \beta}\right| \\
\sigma & =\left|\frac{m_{1}}{m_{2}} e^{-2 i \alpha}\right|
\end{aligned}
$$

as well as the two Majorana CP-violating phases

$$
\alpha=-\frac{1}{2} \arg \left(\frac{K_{3} L_{1}-K_{1} L_{3}}{K_{2} L_{3}-K_{3} L_{2}}\right)
$$




$$
\beta=-\frac{1}{2} \arg \left(\frac{K_{2} L_{1}-K_{1} L_{2}}{K_{3} L_{3}-K_{2} L_{3}} e^{2 i \delta}\right)
$$

The results of Eq. (22), (23),$(24)$ and (25) imply that the two mass ratio $(\rho$ and $\sigma)$ and two Majorana CP-violating phases $(\alpha$ and $\beta)$ are fully determined in terms of three mixing angle and Dirac CP-violating phase $\left(\theta_{12}, \theta_{23}, \theta_{13}\right.$ and $\left.\delta\right)$. The neutrino mass ratios $\rho$ and $\sigma$ are related to the ratios of two neutrino mass-squared ratios obtained from the solar and atmosphere oscillation experiments as

$$
R_{\nu} \equiv \frac{\delta m^{2}}{\left|\Delta m^{2}\right|}=\frac{2 \rho^{2}\left(1-\sigma^{2}\right)}{\left|2 \sigma^{2}-\rho^{2}-\rho^{2} \sigma^{2}\right|}
$$

and to the three neutrino mass as

$$
m_{2}=\sqrt{\frac{\delta m^{2}}{1-\sigma^{2}}} \quad m_{1}=\sigma m_{2} \quad m_{3}=\frac{m_{1}}{\rho}
$$

For normal neutrino mass hierarchy $(\mathrm{NH})$, the latest global-fit neutrino oscillation experimental data, at the $3 \sigma$ confidential level, is list as follows [20]

$$
30.6^{\circ} \leq \theta_{12} \leq 36.8^{\circ}, 35.1^{\circ} \leq \theta_{23} \leq 53.0^{\circ}, 7.5^{\circ} \leq \theta_{13} \leq 10.2^{\circ}
$$

and

$$
\begin{gathered}
6.99 \times 10^{-5} \mathrm{eV}^{2} \leq \delta m^{2} \leq 8.18 \times 10^{-5} \mathrm{eV}^{2} \\
2.19 \times 10^{-3} \mathrm{eV}^{2} \leq \Delta m^{2} \leq 2.62 \times 10^{-3} \mathrm{eV}^{2}
\end{gathered}
$$

For the inverted neutrino mass hierarchy $(\mathrm{NH})$, the differences compared with the $\mathrm{NH}$ are so slight that we shall use the same values given above. It is noted that the global analysis tends to give a $\theta_{23}$ less than $45^{\circ}$ which is

$$
36.2^{\circ} \leq \theta_{23} \leq 42.0^{\circ}
$$

at $2 \sigma$ level and

$$
37.2^{\circ} \leq \theta_{23} \leq 40.0^{\circ}
$$

at $1 \sigma$ level.

As has been pointed out by many papers[21], the $\mu-\tau$ permutation symmetry between $2-3$ rows and $2-3$ columns of $M_{\nu}$ can one patterns to another, i.e

$$
\widetilde{M}_{\nu}=P_{23} M_{\nu} P_{23}
$$


where

$$
P_{23}=\left(\begin{array}{lll}
1 & 0 & 0 \\
0 & 0 & 1 \\
0 & 1 & 0
\end{array}\right)
$$

It is straight to prove that the $\mu-\tau$ permutation symmetry leads to the following relation of mixing parameters between $M_{\nu}$ and $\widetilde{M}_{\nu}$ :

$$
\widetilde{\theta}_{12}=\theta_{12}, \quad \widetilde{\theta}_{13}=\theta_{13}, \quad \widetilde{\theta}_{23}=\frac{\pi}{2}-\theta_{23}, \quad \widetilde{\delta}=\pi-\delta
$$

and $\widetilde{M}_{\nu}$ and $M_{\nu}$ have the same mass eigenvalues. Here we list all the relations of textures related by $\mu-\tau$ symmetry as

$$
\begin{gathered}
A 1 \leftrightarrow A 1, A 2 \leftrightarrow A 8, A 3 \leftrightarrow A 7, A 4 \leftrightarrow A 6, A 5 \leftrightarrow A 5, A 9 \leftrightarrow A 10, B 1 \leftrightarrow C 1 \\
B 2 \leftrightarrow C 7, B 3 \leftrightarrow C 6, B 4 \leftrightarrow C 5, B 5 \leftrightarrow C 4, B 6 \leftrightarrow C 3, B 7 \leftrightarrow C 2, B 8 \leftrightarrow C 10 \\
B 9 \leftrightarrow C 9, B 10 \leftrightarrow C 8, D 1 \leftrightarrow F 2, D 2 \leftrightarrow F 1, D 3 \leftrightarrow F 4, D 4 \leftrightarrow F 3, D 5 \leftrightarrow F 5 \\
D 6 \leftrightarrow F 9, D 7 \leftrightarrow F 8, D 8 \leftrightarrow F 7, D 9 \leftrightarrow F 6, D 10 \leftrightarrow F 10, E 1 \leftrightarrow E 2, E 3 \leftrightarrow E 4 \\
E 5 \leftrightarrow E 5, E 6 \leftrightarrow E 9, E 7 \leftrightarrow E 8, E 10 \leftrightarrow E 10
\end{gathered}
$$

Note that the pattern $A 1, A 5, E 5, E 10$ transforms into itself under the permutation. Thus among the 60 possible textures, only 32 is independent.

\section{NUMERICAL ANALYSIS}

We now present the numerical analysis of the 60 neutrino mass textures of $M_{\nu}$ with one vanishing minor and two equivalent cofactors. Our numerical calculation is performed in the following way:

1)For each pattern, the three mixing angles $\left(\theta_{12}, \theta_{23}, \theta_{13}\right)$ are allowed to vary randomly in their $3 \sigma$ range. Up to now, no bound was set on Dirac CP-violating phase $\delta$, so we vary it randomly in the range of $[0,2 \pi]$.

2)For each group of random numbers $\left(\theta_{12}, \theta_{23}, \theta_{13}, \delta\right)$, we calculate the corresponding two neutrino mass ratios $\rho$ and $\sigma$. Using Eq. (26) , the mass-squared difference ratio $R_{\nu}$ is determined. Then the input parameters is empirically acceptable when 
the $R_{\nu}$ falls inside the the $3 \sigma$ range of experimental data, otherwise they are excluded. Since we have two possible neutrino mass hierarchy, in the analysis we further demand $\rho<\sigma<1$ corresponding to the $\mathrm{NH}$ case and $\sigma<1<\rho$ corresponding to the IH case.

3)For each group of input parameter consistent from the constraint of $R_{\nu}$ above, we randomly generate the value of $\delta m^{2}$ in its $3 \sigma$ range. From Eq. (27) , the three neutrino masses $\left(m_{1}, m_{2}, m_{3}\right)$ are obtained. Given the three neutrino masses, a check shall be made to figure out if the corresponding $\Delta m^{2}$ falls into the $3 \sigma$ range given in (29).

4)Finally, we get the Majorana CP-violating $\alpha$ and $\beta$ though Eq.(24), (25). Since we have already obtained the absolute neutrino mass $m_{1,2,3}$ and $(\alpha, \beta)$, the further constraint from cosmology and $0 \nu 2 \beta$ decay experiment should be considered. A $3 \sigma$ result of $\langle m\rangle_{e e}=(0.11-0.56) \mathrm{eV}$ is reported by the Heidelberg-Moscow Collaboration[22]. However, this result is criticized in Ref [23] and shall be checked by the forthcoming experiment. In this work, we set the upper bound on $\langle m\rangle_{e e}$ at $0.5 \mathrm{eV}$. It is believed that that the next generation $0 \nu \beta \beta$ experiments, with the sensitivity of $\langle m\rangle_{e e}$ being up to $0.01 \mathrm{eV}$, will open the window to not only the absolute neutrino mass scale but also the Majorana-type $\mathrm{CP}$ violation. Besides the $0 \nu \beta \beta$ experiments, a more severe constraint was set from the recent cosmology observation. Recently, an upper bound on the sum of neutrino mass $\sum m_{i}<0.23 \mathrm{eV}$ is reported[5] by Plank Collaboration combined with the WMAP, high-resolution CMB and BAO experiments. Once the input parameter satisfy all the constraint given above, we give a detail discussion on the survived pattern.

A numerical and comprehensive analysis over the sixty patterns of $M_{\nu}$ have been carried out in our study. The main results and the discussion are summarized as follows:

(i) Eight out of the sixty patterns, viz., A2, A3, A5, A7, A8, A9, A10 and E5 are ruled out at $3 \sigma$ confidence level, leaving fifty-two patterns still being compatible with the current experimental data.

(ii) Among the fifty-two surviving patterns, fifteen of them, viz.,D3, D6, D8, E1, 
Table I: The classification of lower bound of $\langle m\rangle_{e e}$

\begin{tabular}{|c|c|}
\hline $0 \mathrm{eV} \sim 0.01 \mathrm{eV}$ & $\begin{array}{c}\mathrm{B} 2-\mathrm{C} 7(\mathrm{NH}), \mathrm{B} 3-\mathrm{C} 6(\mathrm{NH}), \mathrm{B} 8-\mathrm{C} 10(\mathrm{IH}), \mathrm{B} 9-\mathrm{C} 9(\mathrm{NH}), \mathrm{B} 4-\mathrm{C} 5(\mathrm{NH}) \mathrm{D} 2-\mathrm{F} 1(\mathrm{NH}) \\
\text {,D3-F4(NH),D4-F3(NH),D6-F9(NH),D7-F8(NH),E3-E4(NH),E6-E9(NH) } \\
\text { E7-E8(NH), E10-E10(NH),D8-F7(NH),D9-F6(NH),D10-F10(NH) }\end{array}$ \\
\hline $0.01 \mathrm{eV} \sim 0.04 \mathrm{eV}$ & $\begin{array}{c}\text { A1-A1(IH),A4-A6(IH),B1-C1(NH),B5-C4(NH),B6-C3(NH,IH) } \\
\text { B7-C2(NH), B9-C9(IH),B10-C8(NH,IH), D1-F2(NH), D2-F1(IH), } \\
\text { D5-F5(NH),D10-F10(IH) }\end{array}$ \\
\hline $0.04 \mathrm{eV} \sim 0.08 \mathrm{eV}$ & $\begin{array}{c}\text { B1-C1(IH),B2-C7(IH),B3-C6(IH) B4-C5(IH), B5-C6(IH) } \\
\text { B7-C2(IH) D1-F2(IH), D4-F3(IH), D5-F5(IH),D7-F8(IH) } \\
\text { D9-F6(IH),E1-E2(NH) }\end{array}$ \\
\hline
\end{tabular}

E2, E3, E4, E6, E7, E8, E9, E10, F4, F7 and F9 are phenomenological acceptable only for NH spectrum.

(iii) Patterns A4, A6, B8 and C10 are phenomenological acceptable only for IH spectrum.

(iv) The current neutrino oscillation experiments tend to give a $\theta_{23}<45^{\circ}$ result. Using the $2 \sigma$ range given in (30), more patterns of $M_{\nu}$ will be excluded. They are B7, C3, C8, D1, D5 for normal hierarchy and A4, A6, B3, B4, B9, C1, C4, C7, F1, F2, F3, F5, F6, F8, F10 for inverted hierarchy. Hence, the accurate measurement of $\theta_{23}$ and mass hierarchy in future long-baseline neutrino oscillation experiments is essential for our model selection.

(v) The theoretical lower bound of $\langle m\rangle_{e e}$ for patterns are classified in Table. I. The sensitivity of next generation $0 \nu \beta \beta$ experiments is of order $0.01 \mathrm{eV}$, which is just the same order as the $\langle m\rangle_{e e}$ of many allowed patterns we concerned. We expect future $0 \nu \beta \beta$ experiments can set a more severe bound for us to distinguish the allowed texture. It should be emphasized that the whole range of $\langle m\rangle_{e e}$ for textures D6$\mathrm{F} 9(\mathrm{NH})$ and $\mathrm{D} 9-\mathrm{F} 6(\mathrm{NH})$ lie in the range of $[0,0.01]$ which is far from the scope of forthcoming experiment. For other textures, they all have the possibility to be detected in the future experiment. 

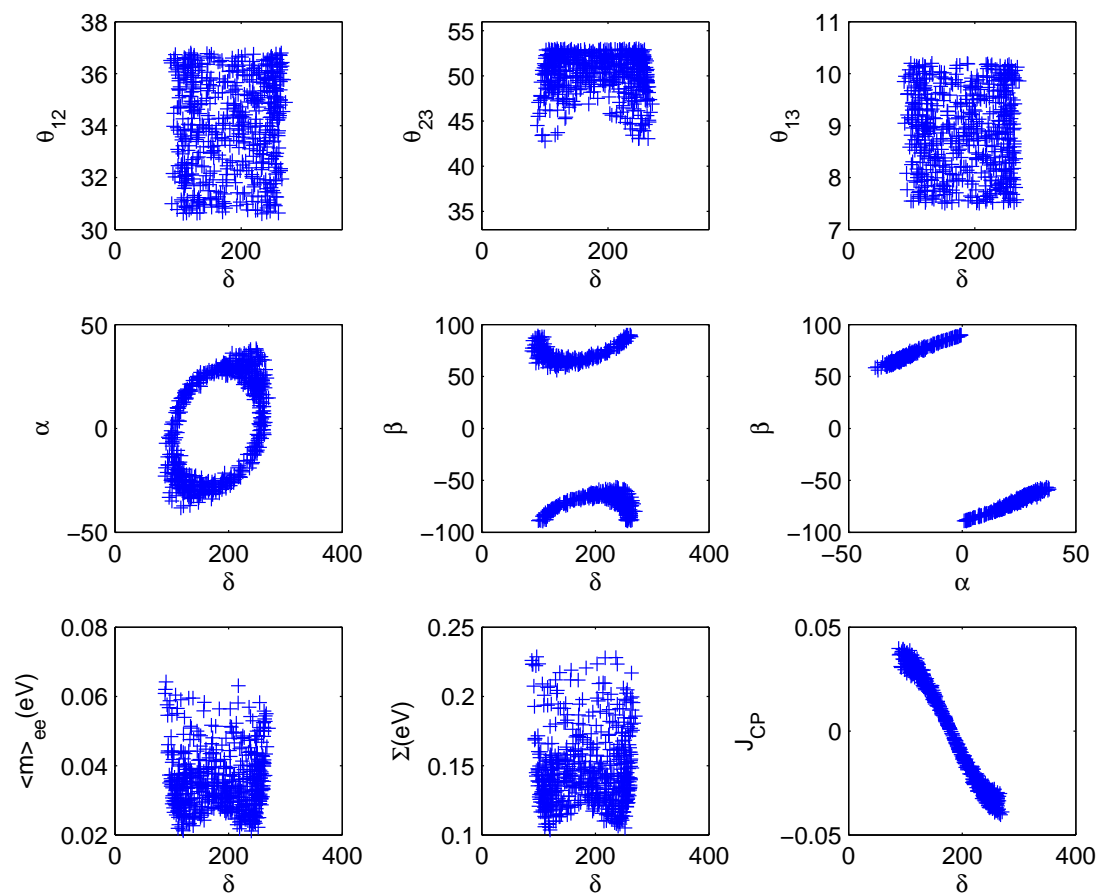

Figure 1: The plots for pattern D1 (NH).

(vi) The upper bound $\Sigma<0.23$ is saturated for most of the surviving patterns except for A4(IH), A6(IH), D3(NH), D6(NH), E3(NH), E6(NH), E7(NH) and E10(NH). On the other hand for all the allowed patterns, $\langle m\rangle_{e e}<0.080 \mathrm{eV}$ is satisfied. Thus compared with the $\langle m\rangle_{e e}<0.5 \mathrm{eV}$ condition, the constraint of $\Sigma$ from cosmology observation is more serve and useful.

A full presentation of the figures for all viable textures will render the paper unreadable. Therefore we present one of the cases, viz., D1 pattern in Fig. 1(for $\mathrm{NH}$ ) and Fig. 2(for $\mathrm{IH})$ as concrete demonstrations.

In both Fig. 1 and Fig. 2, we have plotted the allowed ranges of the mixing angle $\theta_{12}, \theta_{23}$ and $\theta_{13}$ as the function of Dirac-CP violating phase $\delta$ for the D1 pattern in the first row. We have seen that the different type of mass hierarchy shows different feature. For the IH spectrum, although accepted in $3 \sigma$ range, the predicted $\theta_{23}$ is disallowed by $2 \sigma$ range. For the IH spectrum, however, the predicted $\theta_{23}$ is accepted by 

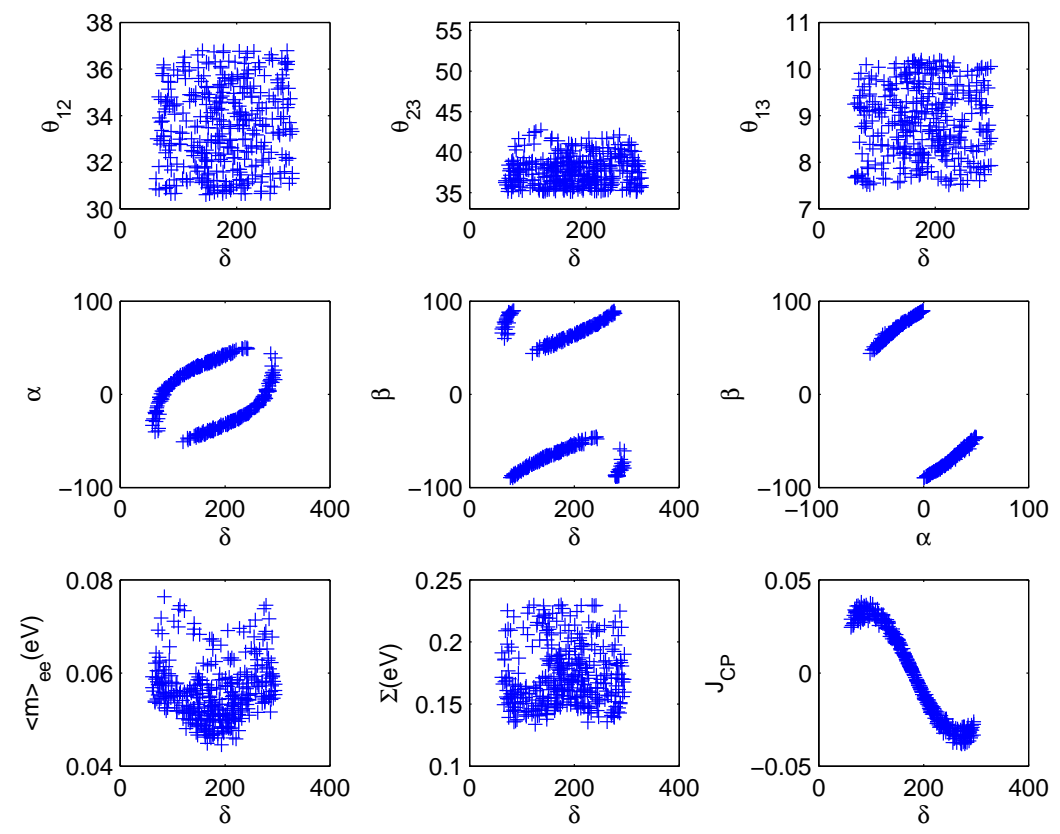

Figure 2: The plots for pattern D1 (IH).

both $3 \sigma$ and $2 \sigma$ ranges. We present the ranges of Majorana-CP violating phase $(\alpha, \beta)$ versus $\delta$ and the $\alpha-\beta$ correlation plots in the second row, where an approximately linear correlation between $\alpha$ and $\beta$ is existed for both $\mathrm{NH}$ and $\mathrm{IH}$ spectrum. In the third row, we have showed the $0 \nu \beta \beta$ decay $\langle m\rangle_{e e}$, the sum of neutrino mass $\Sigma$ and the Jarlskog invariant with respect of $\delta$. One can observe from the figure that the lower bound of $\langle m\rangle_{e e}$ of $\mathrm{D} 1(\mathrm{NH})$ and D1(IH) textures are approximately located at $0.020 \mathrm{eV}$ and $0.045 \mathrm{eV}$, which is in accordance with the results given in Table. I and reachable in the future $0 \nu \beta \beta$ decay experiments. Furthermore, the sum of neutrino masses $\Sigma \geq 0.1 \mathrm{eV}$ are satisfied for both $\mathrm{NH}$ and $\mathrm{IH}$ hierarchy, leading to quasidegenerate $\operatorname{spectrums}\left(m_{i} \approx m_{j} \gg m_{i}-m_{j}\right)$. On the other hand, the Jarlskog invariant is not strongly constrained. The Jarlskog invariant can reach its maximal value, $\left|J_{C P}\right| \sim 4 \%$ if the Dirac-CP phase $\delta$ is close to $\pi / 2$ and $3 \pi / 2$ for normal as well as inverted hierarchy. 


\section{SYMMETRY REALIZATION}

It is generally believed that the observed neutrino mixing pattern suggests some underlying discrete flavor symmetries (for a review, see[24]). It has been proved that the hybrid $M_{\nu}$ textures can be achieved by imposing the $S_{3} \otimes Z_{3}$ flavor symmetry in type-II seesaw model[9]. Thus, it is natural to ask if the hybrid $M_{\nu}^{-1}$ textures we studied can arise from this flavor symmetry. As an illustration, We propose a flavor model for D4 pattern. Consider the three left-handed neutrino fields $\nu_{i L}$, three right-handed neutrino fields $\nu_{R i}$ where $i=e, \mu, \tau$ in the following representation of $S_{3} \otimes Z_{3}$ group

$$
l_{\mu L} \sim\left(1, \omega^{2}\right), \quad\left(\begin{array}{c}
l_{e L} \\
l_{\tau L}
\end{array}\right) \sim(2, \omega), \quad \nu_{\mu R} \sim(1, \omega), \quad\left(\begin{array}{c}
\nu_{e R} \\
\nu_{\tau R}
\end{array}\right) \sim\left(2, \omega^{2}\right)
$$

and for scalar fields

$$
\left(\begin{array}{l}
\chi_{1} \\
\chi_{2}
\end{array}\right) \sim(2,1), \quad\left(\begin{array}{l}
\chi_{3} \\
\chi_{4}
\end{array}\right) \sim\left(2, \omega^{2}\right), \quad \chi_{5} \sim\left(1, \omega^{2}\right), \quad \Phi \sim(1,1)
$$

where $\omega=e^{i 2 \pi \backslash 3}$ Then the Lagrangian corresponding to the Dirac neutrino mass term and Majorana neutrino mass term are given by

$$
\begin{array}{r}
L_{D}=Y_{1}\left(\bar{l}_{\mu L} \nu_{\mu R}\right) \Phi+Y_{2}\left(\bar{l}_{e L} \nu_{e R}+\bar{l}_{\tau L} \nu_{\tau R}\right) \Phi+h . c \\
L_{M}=\frac{1}{2} y_{1} \nu_{\mu R}^{T} C^{-1}\left(\chi_{1} \nu_{e R}+\chi_{2} \nu_{\tau R}\right)-\frac{1}{2} y_{2}\left(\nu_{e R}^{T} C^{-1} \nu_{e R}+\nu_{\tau R}^{T} C^{-1} \nu_{\tau R}\right) \chi_{5} \\
-\frac{1}{2} y_{3}\left[\left(\nu_{e R}^{T} C^{-1} \nu_{\tau R}+\nu_{\tau R}^{T} C^{-1} \nu_{e R}\right) \chi_{3}+\left(\nu_{e R}^{T} C^{-1} \nu_{e R}-\nu_{\tau R}^{T} C^{-1} \nu_{\tau R}\right) \chi_{4}\right]+h . c
\end{array}
$$

where the direct product $2 \otimes 2=1+1^{\prime}+2$ for $S_{3}$ group is used[25]. When the Higgs fields acquire the vacuum expectations values $(\mathrm{VEVs})\langle\Phi\rangle=v,\left\langle\chi_{i}\right\rangle=v_{i}$, we obtain the Dirac mass matrix and the Majorana mass matrix as

$$
M_{D}=\left(\begin{array}{ccc}
Y_{2} v & 0 & 0 \\
0 & Y_{1} v & 0 \\
0 & 0 & Y_{2} v
\end{array}\right), \quad M_{R}=\left(\begin{array}{ccc}
y_{2} v_{5}+y_{3} v_{4} & y_{1} v_{1} & 2 y_{3} v_{3} \\
y_{1} v_{1} & 0 & y_{1} v_{2} \\
2 y_{3} v_{3} & y_{1} v_{2} & y_{2} v_{5}-y_{3} v_{4}
\end{array}\right)
$$

Once the $v_{4}=0$ after the scalar potential is minimized, one can check that the neutrino mass matrix $M_{\nu}=M_{D} M_{R}^{-1} M_{D}^{T}$ satisfies the condition $C_{22}=0$ and $C_{11}=C_{33}$ of D4 pattern. 
On the other hand, the charged lepton mass matrix $M_{l}$ should be diagonal. The right-handed charged lepton are assigned as

$$
\mu_{R} \sim\left(1, \omega^{2}\right), \quad e_{R} \sim\left(1^{\prime}, \omega\right), \quad \tau_{R} \sim(1, \omega)
$$

To generate the charged lepton mass, we still need a standard Higgs field which is a singlet scalar under the flavor symmetry and scalar doublet fields $\Phi_{1}$ and $\Phi_{2}$

$$
H \sim(1,1), \quad\left(\begin{array}{l}
\Phi_{1} \\
\Phi_{2}
\end{array}\right) \sim(2,1),
$$

The Lagrangian of charged lepton sector is given by

$$
L_{l}=Y_{\mu} \bar{l}_{\mu L} \mu_{R} H+Y_{e}\left(\bar{l}_{e L} \Phi_{2}-\bar{l}_{\tau L} \Phi_{1}\right) e_{R}+Y_{\tau}\left(\bar{l}_{e L} \Phi_{1}+\bar{l}_{\tau L} \Phi_{2}\right) \tau_{R}+\text { h.c }
$$

After the vacuum expectation $\langle H\rangle=v_{6},\left\langle\Phi_{2}\right\rangle=v_{7}$ and $\left\langle\Phi_{1}\right\rangle=0$ are taken, we obtain the charged lepton mass matrix $M_{l}=\operatorname{Diag}\left\{m_{e}, m_{\mu}, m_{\tau}\right\}$ with $m_{e}=Y_{e} v_{7}, m_{\mu}=Y_{\mu} v_{6}$, $m_{\tau}=Y_{\tau} v_{7}$

Hence we construct the a hybrid $M_{\nu}^{-1}$ neutrino mass texture via $S_{3} \otimes Z_{3}$ flavor symmetry. However, it should be stressed that there still remain two things unsolved: 1), it is not trivial to guarantee the Higgs fields are broken to a specific direction. i.e. the vacuum alignment problem; 2). As similar as the hybrid $M_{\nu}$ ones, not all the textures can be realized by $S_{3} \otimes Z_{3}$ flavor symmetry. However, tt is noted that in another work[26], the $Z_{2} \otimes Z_{4}$ symmetry is produced to generate the texture

$$
\left(\begin{array}{ccc}
0 & \triangle & \triangle \\
\triangle & \times & \times \\
\triangle & \times & \times
\end{array}\right)
$$

which is just the A1 pattern.

In this sense, the symmetry realization for all neutrino mass patterns in a systematic and self-consistent way deserves further research. 


\section{CONCLUSION AND DISCUSSION}

In this work, we have presented a numerical and comprehensive study of the neutrino mass textures with one vanishing minor and two equal cofactors. Among the sixty texture, only eight of them are ruled out for $\mathrm{NH}$ as well as $\mathrm{IH}$ spectrum by the current experiments data at $3 \sigma$ level. The neutrinoless double decay experiments and the neutrino sum mass from cosmology observation are also discussed. We expect that further experiment will provide us more accurate determination on the mixing angle, CP-violating phase, mass hierarchy and neutrino absolute mass, and will finally help us select the appropriate structure of mass texture. In this processes, the determination of $\theta_{23}$ plays an essential role on the model selection. A flavor realiza-

tion based on $S_{3} \otimes Z_{3}$ symmetry is also discussed. The symmetry realization of all the textures in a systematic and self-consistent way deserves further research. We except that a cooperation between theoretical study from the flavor symmetry point view and a phenomenology study will help us reveal the structure of neutrino mass texture.

\section{Acknowledgments}

The author would like to thank S. Dev, Zheng-Mao Sheng, Jia-Hui Huang, Ji-Yuan, Liu, and Shun Zhou for the useful discussion during this work.

[1] Q.R. Ahmad et al.(SNO Collaboration), Phys. Rev. Lett 89, 011301(2002); K. Eguchi et al. (KamLAND Collaboration), Phys. Rev. Lett 90, 021802(2003); M.H. Ahn et al. (K2K Collaboration), Phys. Rev. Lett 90, 041801(2003).

[2] F.P. An et al. (DAYA-BAY Xollaboration), Phys. Rev. Lett. 108, 171803(2012).

[3] J.K. Ahn et al. (RENO Collaboration), Phys. Rev. D108, 191802(2012).

[4] G. Hinshaw et al. (WMAP Collaboration), arXiv: 1212.5226.

[5] P.A.R. Ade et al. (Planck Collaboration), arXiv: 1303.5076. 
[6] S.M. Bilenky and C. Giunti, Mod. Phys, Lett. A16, 1230015(2012).

[7] P.H. Frampton, S. L. Glashow, and D. Marfatia, Phys. Lett. B536, 79(2002); Z,-z. Xing, Phys. Lett. B530, 159(2002); M. Randhawa, G. Ahuja, and M. Gupta, Phys. Lett. B643, 175(2006); A. Merle, and W. Rodejohann, Phys. Rev. D73, 073012(2006); S. Dev, S. Kumar, S. Verma, and S. Gupta, Phys. Rev. D76, 013002(2007); S. Dev, S. Kumar, S. Verma, and S. Gupta, Nucl. Phys. B784, 103(2007); G. Ahuja, S. Kumar, M. Randhawa, M. Gupta, and S. Dev, Phys. Rev. D76, 013006(2007); S. Dev, S. Kumar, Mod. Phys, Lett. A22, 1401(2007); S. Kumar, Phys. Rev. D84, 077301(2011); P.O. Ludl, S. Morisi, and E. Peinado, Nucl. Phys. B857, 411(2012); W. Grimus. and P.O. Ludl, arXiv:1208.4515; D. Meloni, and G. Blankenburg, Nucl. Phys. B867, 749(2013); H. Fritzsch, Z.-z. Xing, and S. Zhou, J. High Energy Phys. 09 (2011)083.

[8] S. Kaneko, H. Sawanaka, and M. Tanimoto, J. High Energy Phys. 08 (2005)073; S. Dev, S. Verma, and S. Gupta, Phys. Lett. B687, 53(2010); S. Dev, S. Gupta, and R.R. Gautam, Phys. Rev. D82, 073015(2010); S. Goswami, S. Khan, and A. Watanable, Phys. Lett. B687, 53(2010), W. Grimus, and P. O. Ludl, arXiv: 1208.4515.

[9] J.-Y. Liu and S. Zhou, Phys. Rev. D87, 093010(2013).

[10] X.-G. He and A. Zee, Phys. Rev. D68, 037302(2003).

[11] G.C. Branco, R. Gonzalez Felipe, F.R. Joaquim, and T. Yanagida, Phys. Lett. B562, 265(2003); B.C. Chauhan, J. Pulido, and M. Picariello, Phys. Rev. D73, 053003(2006).

[12] L. Lavoura, Phys. Lett. B609, 317(2005); E.I. Lashin and N. Chamoun, Phys. Rev. D78, 073002(2008); E.I. Lashin and N. Chamoun, Phys. Rev. D80, 093004(2009); S. Dev, S. Verma, S. Gupta, and R.R. Gautam, Phys. Rev. D81, 053010(2010); S. Dev, S. Gupta, and R.R. Gautam, Mod. Phys, Lett. A26, 501(2011); S. Dev, S. Gupta, R.R. Gautam, and L. Singh, Phys. Lett. B706, 168(2011); T. Araki, J. Heeck, and J. Kubo, J. High Energy Phys. 07 (2012)083; S. Verma, Nucl. Phys. B854, 340(2012).

[13] H.A. Alhendi, E.I. Lashin, and A.A. Mudlej, Phys. Rev. D77, 013009(2008).

[14] S. Dev, R.R. Gautam, and L. Singh, Phys. Rev. D87, 073011(2013).

[15] H. Fritzsch, M. Gell-Mann, and P. Minkowski, Phys. Lett. B59, 256(1975); P. Minkowski, Phys. Lett. B67, 421(1977); T. Yanagida, in Proceedings of Workshop 
on Unified Theory and the Baryon Number of the Universe, edited by O. Sawada and A. Sugamoto(KEK, Tsukuba, 1979), p. 95; M. Gell-Mann, P. Ramond, and Slansky, in Supergravity, edited by P. van. Nieuwenhuizen and D.Z. Freeman (North-Holland, Amsterdam,1979), p. 315; R.N. Mohapatra and G. Senjanovic, Phys, Rev. Lett. 44, 912(1980); J. Schechter and J. W. F. Valle, Phys. Rev. D22, 2227(1980); J. Schechter and J. W. F. Valle, Phys. Rev. D25, 774(1982).

[16] E. Ma, Phys. Rev. D71, 111301(2005).

[17] G.L. Fogli, E. Lisi, A. Marrone, and A. Palazzo, Prog. Part. Nucl. Phys. 57, 742(2006).

[18] B. Pontecorvo, Zh. Eksp. Teor. Fiz. 33, 549(1957); Z. Maki, M. Nakagawa, and N. Sakata, Prog. Theor. Phys. 28, 870(1962).

[19] C. Jarlskog, Phys, Rev. Lett. 55, 1039(1985).

[20] G.L. Fogli, E. Lisi, A. Marrone, D. Montanino, A. Palazzo, and A.M. Rotunno, Phys. Rev. D86, 013012(2012); D. V. Forero, M. Tortola, and J. W. F. Valle, Phys. Rev. D86, 073012(2012); M. C. Gonzalez-Garcia, Michele Maltoni, Jordi Salvado, and Thomas Schwetz, J. High Energy Phys. 12 (2012)123.

[21] T. Fukuyama and H. Nishiura, arXiv: 9702253; R. N. Mohapatra and S. Nussinov, Phys. Rev. D60, 013002, (1999); E. Ma and M. Raidal, Phys. Rev. Lett. 87, 011802 (2001); C. S. Lam, Phys. Lett. B507, 214 (2001); K. R. S. Balaji, W. Grimus and T. Schwetz, Phys. Lett. B508, 301 (2001); W. Grimus and L. Lavoura, Acta Phys. Pol. B32, 3719 (2001).

[22] H.V. Klapdor-Kleingrothaus, A. Dietz, H.L. Harney, and I.V. Krivosheina, Mod. Phys. Lett. A16, 2409(2001).

[23] C.E. Aalseth et al. Mod. Phys. Lett. A17, 1475(2002); F. Feruglio, A. Strumia, and F. Vissani, Nucl. Phys. B637, 345(2002).

[24] G. Altarelli and F. Feruglio, Rev. Mod. Phys. 82, 2701(2010); S. K. King and C, Luhn, Rept.Prog.Phys. 76 (2013) 056201.

[25] H. Ishimori, T. Kobayashi, H. Ohki, Y. Shimizu, H. Okada, and M. Tanimoto, Prog. Theor. Phys. Suppl. 183 1(2010).

[26] S. Dev, R. R. Gautam, L. Singh, arXiv: 1306.4281. 